DOI: $10.21767 / 2471-8505.100095$

\section{Commentary on 'Application of the Cultural Competence Model in the Experience of Care in Nursing Professionals Primary Care'}

Received: August 07, 2017; Accepted: August 23, 2017; Published: August 30, 2017

\section{María Dolores Gil Estevan ${ }^{1 *}$ and María del Carmen Solano Ruíz² \\ 1 Departamento de Salud de Elda, HGU de Elda, Alicante, Spain \\ 2 Departamento de Enfermería, Facultad de Ciencias de la Salud, Universidad de Alicante, Alicante, Spain}

\author{
*Corresponding author: \\ María Dolores Gil Estevan \\ Iola.gilesteban@gmail.com \\ Departamento de Salud de Elda, HGU de Elda, \\ Alicante, Spain.
}

\author{
Citation: Gil Estevan MD, del Carmen \\ Solano Ruíz M (2017) Commentary on \\ 'Application of the Cultural Competence \\ Model in the Experience of Care in \\ Nursing Professionals Primary Care'. \\ J Intensive \& Crit Care Vol. 3 No. 3:36
}

The language barrier hinders not only communication, but also the monitoring and control of the health-disease process, so it is vital to be culturally competent in the application of care based on the cultural characteristics individual or community and the promotion cultural comprehension of the different human situations.

The authors emphasize the importance of acquiring knowledge, skills and attitudes towards cultural diversity to provide culturally competent care. The nurse is the main communicating agent and interpreter of the reality of patients.

On the other hand, they conclude that the application of Purnell's model allows the identification of the experiences of nurses in the provision of culturally competent care with an ethnographic focus.

The perceptions and experiences of nurses in providing care to these women provide valuable information and allow identifying the factors that influence the relationship with the patients. This will allow us to elaborate Strategies that contribute to provide

quality care based on cultural competence.

In the original article "Application of the cultural competence model in the experience of care in nursing professionals Primary
Care", published by the Atención Primaria magazine, the authors, María Dolores Gil Estevan and María del Carmen Solano Ruíz, conduct a qualitative investigation of Phenomenological carácter.
The objective is to know the experiences and the perception of the nursing professionals in the provision of care and health
promotion to women at risk of social vulnerability. The study applies the model of cultural competence of Larry Purnell in gypsies women and women from Morocco and Romania [1].
Faced with the provision of care to an increasingly multicultural society, difficulties arise related to socio-cultural factors that influence the relationship between professionals and users of the system. Subtle racism and historical prejudices create uncomfortable and distrustful situations.
The method used consisted in the analysis of focus groups, semistructured interviews and field notes, using as reference the 12 domains of the model of cultural competence of Larry Purnell and taking into account the assumptions of anthropology and phenomenology.

After analyzing the information obtained, we conclude the need to implement strategies to improve the delivery of culturally competent care;

$\checkmark$ Development of training programs in cultural competence for professionals.

$\checkmark$ Training professionals in the management of social inequalities in health.

$\checkmark$ Design of specific programs and protocols for the social group to be served.

$\checkmark$ Promotion of research in groups at risk of social vulnerability. 


\section{References}

1 Gil Estevan MD, Solano Ruíz MC (2017) Application of the cultural

competence model in the experience of care in nursing professionals primary care. Aten Primaria pii: S0212-6567(16)30247-5. 